\title{
Conceptual Design of a Nanosatellite Incubator Using Axiomatic Design and a Mechatronic Multicriteria Profile
}

\author{
Yuanchao $\mathrm{Ma}^{1}$, Aurelian Vadean ${ }^{1}$, Giovanni Beltrame ${ }^{1}$, Sofiane Achiche ${ }^{1}$ \\ ${ }^{1}$ École Polytechnique de Montréal
}

\begin{abstract}
The CubeSat platform has become increasingly popular in recent decades. Due to its relatively low research cost and short development time, it is often used to conduct experiments in space in various fields. Since a satellite payload is usually a cross-disciplinary system, one of its design challenges is how to optimize the dependencies and correlations among components while ensuring customer-specific mission requirements. Based on these points, this paper presents a conceptual design of a one unit $(1 \mathrm{U}-10 \mathrm{~cm} \times 10 \mathrm{~cm} \times 10 \mathrm{~cm})$ miniaturized incubator for a nanosatellite mission using Axiomatic Design and a Mechatronic Multicriteria Profile. During this design process, 64 concepts were derived and evaluated, resulting in an "Elite" candidate with a rating of $0.89 / 1$. The concept generated is a pressurized microincubator consisting of four subsystems: cultivation system, temperature control system, supply system, and monitoring system. Two prototypes designed and manufactured based on this concept have successfully completed for plant growth experiments in terrestrial conditions.
\end{abstract}

Keywords: Conceptual Design, Axiomatic Design, Mechatronic Multicriteria Profile, CubeSat

\section{Introduction}

Space biology is the combination of space technology and biological research to understand the behavior of organisms during space flight. The effects of microgravity and radiation are important factors to consider for space experiments compared to the ground environment. And with the growing interest in deep space exploration and commercial human spaceflight, there is a need to develop more advanced life support systems. In addition, improving our understanding of how plant cultivation is affected by microgravity is critical to developing the ability to produce food off Earth and planning long-term missions (Häuplik-Meusburger et al., 2011; Zabeau et al., 2016).

Traditionally, only a few government-supported institutions in the space industry have been able to design and build large, sophisticated satellites with large budgets to conduct relevant experiments. However, in the last decade, the space industry has shown a growing interest in small satellites with smaller sets of instruments (or individual sensors) from an operational and technical point of view (Poghosyan and Golkar, 2017). CubeSats provide a low-cost means to study scientific phenomena in the universe and to advance new engineering concepts. The relatively reduced cost of participating in space activities has encouraged governments, industry, and educational institutions to develop small satellite programs (Woellert et al., 2011). As a result, the development of CubeSat has accelerated significantly in recent years (Boone et al., 2014; Hegde et al., 2019; Xiang et al., 2014; 
Conceptual Design of a Nanosatellite Incubator Using Axiomatic Design and a Mechatronic Multicriteria Profile

Zabeau et al., 2016). In addition, advances in miniaturization of commercial off-the-shelf (COTS) device in various fields such as telecommunications, electronics, sensors, and instrumentation have stimulated the development of small space missions based on the CubeSat standard (Lee et al., 2009). These advances have allowed payloads to perform a variety of measurements and experiments on CubeSat with many small COTS autonomous instruments and systems (Kramer and Cracknell, 2008).

The paper describes a research work involving the conceptual design of an intelligent automated miniaturized incubator system that can perform experiments and autonomously cultivate plants on the CubeSat platform. First, given the specific experimental goals of the satellite payload, it was particularly important to meet the customized design requirements needed for it. (Kulak et al., 2010) stated that there is a wide variety of customer requirements that influence decision makers to find the most suitable alternative in many product and system design decisions. Therefore, the accurate interpretation of customer requirements into specific design parameters is a major challenge for design tasks. To address this problem, this paper firstly uses the Axiomatic Design (AD) approach to obtain different feasible solutions for further evaluation and decision-making. The next step then involves the complex task of space system design. Both (Lubián-Arenillas et al., 2019) and (Hegde et al., 2019) mentioned that during the CubeSat design process, subsystems are usually designed simultaneously, while considering the compatibility between subsystems and their synergistic effects. In addition, nanosatellite missions usually consist of small teams and their pre-operational phase is usually short. It follows that it is one of the important elements to identify and correct errors earlier in the design process, thus reducing the overall design time (Lubián-Arenillas et al., 2019). In summary, this is the step where we need a fast, concise, and efficient method, while taking into account the coupling between the subsystems in a well-thought-out way, in order to obtain a reliable and optimal solution. Moreover, give that it covers COTS components from various disciplines such as electrical engineering, mechanical engineering, software engineering and control engineering (ESA, 2016), and such a system also meets the definition of mechatronic systems by (Rzevski, 2014). We therefore chose the Mechatronic Multicriteria Profile (MMP) including five main criteria (Machine Intelligence Quotient, Reliability, Complexity, Flexibility, and Cost of manufacturing and production) proposed by (Mohebbi et al., 2014, 2018) for mechatronic concept evaluation in the conceptual design phase. In the decision phase, a quantitative evaluation method is used to generate evaluation index by fuzzy measures and Choquet Integral to rank the generated feasible concepts and select the "Elite" set among the design solutions.

This paper is structured as follows. Section 2 describes the design methodology and process, followed by Section 3, which presents the final concept and results of the ground tests obtained through the proof-of-concept product. Finally, we conclude the work in Section 4.

\section{Design methodology and process}

Figure 1 illustrates the general idea of the method, and each step of the method is described in detail in this section. After identifying the design requirements, the whole approach starts by generating the different design alternatives by respecting the two axioms of AD. Then 
the different concepts are evaluated separately for each of the five sub-criteria according to the MMP developed by (Mohebbi et al., 2014, 2018). Finally, the Global Concept Score (GCS) of each solution are obtained by fuzzy measures and Choquet Integral, and the optimal solution is obtained after comparison.

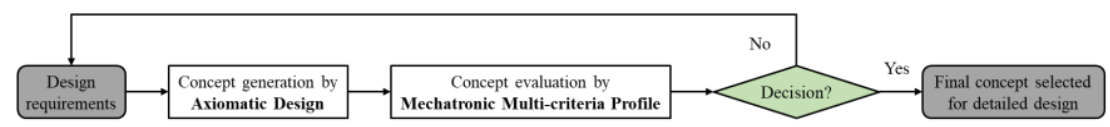

Figure 1 The proposed evaluation method

\subsection{Concept generation by Axiomatic Design}

Axioms are general principles which are obvious practical situations and cannot be proven correct, but they have no counter examples (Suh and Sekimoto, 1990). In 1990, Axiomatic Design was proposed by (Suh, 1990) and since then it has been widely used to solve complex design problems, especially in preliminary conceptual phase. AD provides a systematic approach to implement scientific solutions that respect both independence axiom and information axiom. The design process is referred to as a hierarchical mapping from a high level of abstraction to a more concrete and appropriate solution. In AD, there are four distinct design domains: Customer Attributes (CA), Functional Requirements (FR), Design Parameters (DP), and Process Variables (PV). PV would not be considered in this article since we are only focusing on the conceptual design of a mechatronic system.

An accurate analysis of design requirements has a considerable impact on the definition of design parameters and the choice of alternatives. The first step in AD is to coordinate and translate all design requirements into CAs. Our design requirements include design constraints as well as experimental parameters. Since our incubator will be a one unit (1U) CubeSat payload, the relevant constraints are indicated in CA1 in Table 1 . The experimental specifications and the experimental protocol have been clearly stated in (Ma, 2018). Here we translate them into CA2 in Table 1 . To ensure successful germination and growth of plants, parameters such as air and pressure, nutrient solution, illumination, and temperature must be maintained during the experiment. In addition, the environmental parameters inside the incubator need to be monitored for further analysis of the entire growth process.

Figure 2.a shows the AD domains involved in this design. Constraints in AD represent the bounds on an acceptable solution (Suh, 1990). Thus, CA1.X are interpreted as constraints Cs on FRs and DPs, and the subsequent analysis process and the final generated concepts must respect these constraints Cs. Then, the definition of the first level of FRs is completed by an injective transformation from CA2 to FRs. The application of the first axiom can then be described in terms of a Design Matrix (DM) corresponding to the relationship between FRs and DPs at the same level:

$$
\left\{F R_{S}\right\}=[D M]\left\{D P_{S}\right\}: \quad \quad \text { Uncoupled }:[D M]=\left[\begin{array}{cc}
X & 0 \\
0 & X
\end{array}\right]
$$

Decoupled: $[D M]=\left[\begin{array}{ll}X & 0 \\ X & X\end{array}\right] ; \quad$ Coupled: $[D M]=\left[\begin{array}{ll}X & X \\ X & X\end{array}\right]$. 
Conceptual Design of a Nanosatellite Incubator Using Axiomatic Design and a Mechatronic Multicriteria Profile

Table 1 Design requirements: CAs of $\mathrm{AD}$

\begin{tabular}{|c|c|c|c|}
\hline \multicolumn{2}{|c|}{ CAs } & Description & Details \\
\hline CA1 & $=$ Cs & Design constraints & $1 \mathrm{U}$ CubeSat \\
\hline & CA1.1 & Maximum volume & $86 \mathrm{~mm} \times 97 \mathrm{~mm} \times 97 \mathrm{~mm}$ \\
\hline & CA1.2 & Maximum weight & $1.33 \mathrm{~kg}$ \\
\hline & CA1.3 & Maximum power & $2.5 \mathrm{~W}$ \\
\hline & CA1.4 & Maximum operating voltage & $3.3 \mathrm{~V}$ \\
\hline & CA1.5 & Preferred sensor interface & I2C \\
\hline CA2 & & Experimental parameters & Plant growth experiment \\
\hline & CA2.1 & Air and pressure & $100 \mathrm{KPa} \pm 10 \%$ \\
\hline & CA2.2 & Nutrient solution & $\begin{array}{c}\text { Flow rate: } 0.15 \mathrm{ml} / \mathrm{s} \text { and } \\
0.015 \mathrm{ml} / \mathrm{s}\end{array}$ \\
\hline & CA2.3 & Illumination & $200 \mu \mathrm{mol} /\left(\mathrm{m}^{2} \cdot \mathrm{s}\right)$ \\
\hline & CA2.4 & Temperature & $26^{\circ} \mathrm{C} \pm 2{ }^{\circ} \mathrm{C}$ \\
\hline & CA2.5 & Parameters to be monitored & $\begin{array}{c}\mathrm{CO}_{2}, \mathrm{O}_{2}, \text { pressure, } \\
\text { humidity, temperature }\end{array}$ \\
\hline
\end{tabular}

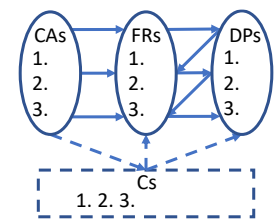

(a) Design domains involved in this study

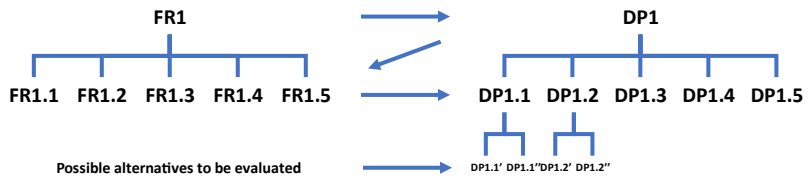

(b) Different levels between FRs and DPs

Figure 2 Axiomatic Design: consecutive mapping between subdomains

A concept with low coupling (uncoupled or decoupled) will be preferred. As shown in Figure 2.b, we can then follow the independence axiom to derive the relevant subsystems and components with low coupling from the continuous mapping between FRs and DPs. Also, (Tang et al., 2009) pointed out that for an FR there may be more than one corresponding DP and several solutions may satisfy the functional independence axiom. This paper is a good example of multiple available COTS products for the same DP. We can see from Figure 2.b that DP1.1 can have both DP1.1' and DP1.1" as possible products to choose from. And the combination of these different DP alternatives with each other becomes the conceptual design solution that needs to be evaluated by MMP.

The complete derivation process and results of the AD of our design process can be found in the work presented in (Ma, 2018). The next step is to use the results obtained from AD to form the concept. The generation of design alternatives was performed after the designer carefully identified feasible COTS devices that respect all the constraints Cs previously identified, as shown in Figure 3. However, some subcomponents have no alternatives (e.g., fans) or were preselected (e.g., ceramic tubes and soil pack for the experiment; camera, housing material PEEK and EPS for satellite) and some would be customized (e.g., PCB, container, mechanical condenser). Therefore, these components are not considered when using the MMP method. All optional COTS alternatives are shown in the "Alternatives" row. With these alternatives, we have been able to generate 64 concepts to evaluate. In the 
next paragraphs, in order to simplify the presentation, we have chosen to study 4 concepts shown in Table 2 to demonstrate the MMP approach.

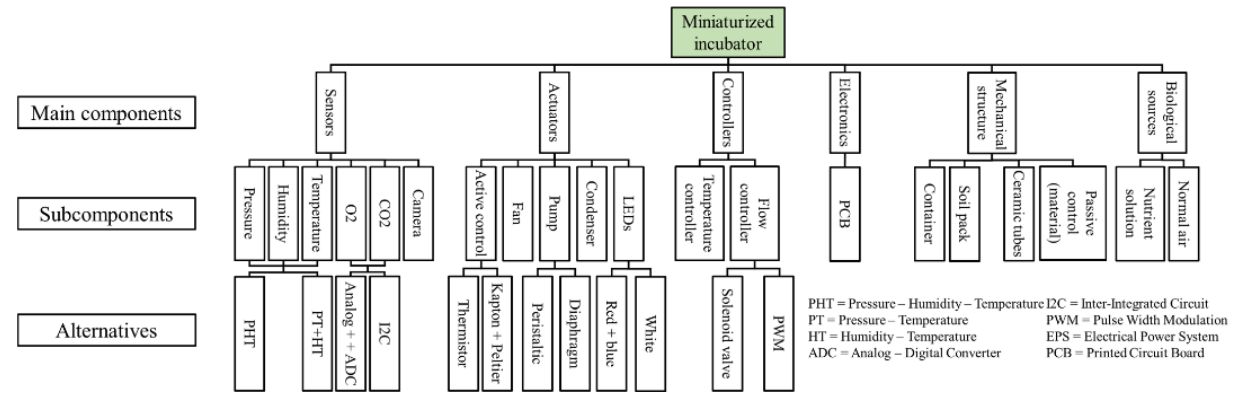

Figure 3 Concepts generated by $\mathrm{AD}$ and possible alternatives to be developed by MMP

Table 2 Four concepts to be evaluated with MMP

\begin{tabular}{|c|c|c|c|c|}
\hline & Concept 1 & Concept 2 & Concept 3 & Concept 4 \\
\hline Gas sensors & Analog+ADC & I2C & I2C & Analog+ADC \\
\hline $\begin{array}{c}\text { Environmental } \\
\text { sensors }\end{array}$ & PT+HT & PHT & PHT & PT+HT \\
\hline $\begin{array}{c}\text { Thermal } \\
\text { module }\end{array}$ & $\begin{array}{c}\text { Kapton }+ \\
\text { Peltier }\end{array}$ & Thermistors & $\begin{array}{c}\text { Kapton }+ \\
\text { Peltier }\end{array}$ & Thermistors \\
\hline Pump & Peristaltic & Diaphragm & Diaphragm & Peristaltic \\
\hline LED & White & Red+Blue & White & Red+Blue \\
\hline Flow controller & Solenoid valve & Solenoid valve & PWM & PWM \\
\hline
\end{tabular}

\subsection{Concept evaluation by Mechatronic Multicriteria Profile}

After all possible alternatives have been obtained using AD, the next task is to determine the "Elite" design by comparing the different sets of components. An optimal mechatronic design requires an accurate and systematic design evaluation step. Obtaining the best solution is a very complex task if the performance parameters involved are not determined and their joint impact considered. And this process includes comparison and decisionmaking (Ullman, 2010). In order to form an integrated and systematic evaluation approach, (Mohebbi et al., 2018) identified the most important criteria and their associated subcriteria to form an index vector called MMP:

$M M P=[M I Q R S C X F X C T]^{T}$

It is composed of five normalized elements including machine intelligence quotient (MIQ), system reliability (RS), design complexity $(\mathrm{CX})$, system flexibility (FX) and production cost (CT). Figure 4 depicts the MMP, and all the corresponding sub-criteria used in this design. Specifically, the Fault Tree Analysis (FTA) approach is combined with the MMP in this paper for the system reliability, as spatial systems require a critical level of reliability analysis(Bidner, 2010; Stamatelatos et al., 2002). Numerical determinations of the rest criteria and their sub-criteria are calculated with reference to the original MMP method. 
Conceptual Design of a Nanosatellite Incubator Using Axiomatic Design and a Mechatronic Multicriteria Profile

We define $x_{i}$ as the parameters used in calculating a criterion or a sub-criterion $i$. The normalized value of the criterion $x_{i}{ }^{*}$ is calculated by function $f()$ :

$x_{i}{ }^{*}=f\left(x_{i}\right)=\left\{\begin{array}{lr}\frac{x_{i}}{\max \left(x_{i}\right)} & \text { if a larger } x_{i} \text { is more desired, e.g., } M I Q, R S, F X \\ \frac{1}{\left(\frac{x_{i}}{\min \left(x_{i}\right)}\right)} & \text { if a smaller } x_{i} \text { is more desired, e.g., } C X, C T\end{array}\right.$

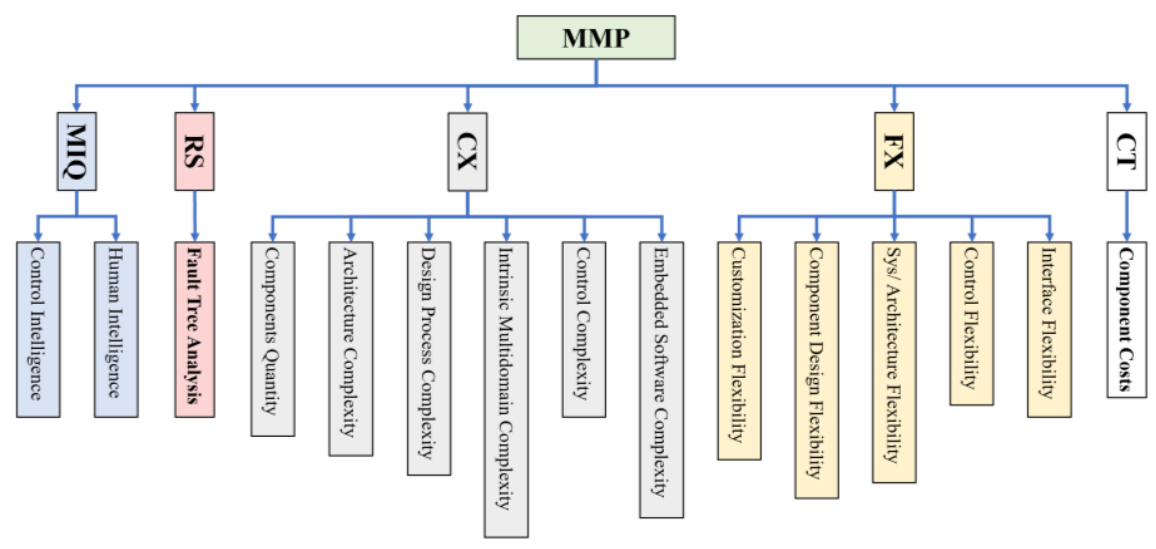

Figure $4 \mathrm{MMP}$ and all sub-criteria

After determining and normalizing each sub-criterion, the value of each major criterion is evaluated by linear summation using a weighting factor as follows:

$m_{i}=\sum_{j=1}^{n} \omega_{j} \rho_{i}^{*}$

where $\rho_{i}{ }^{*}$ is the normalized value of each sub-criterion, $n$ is the total number of subcriteria, and $\omega_{j}$ is the weight assigned by the designer associated with each sub-criterion.

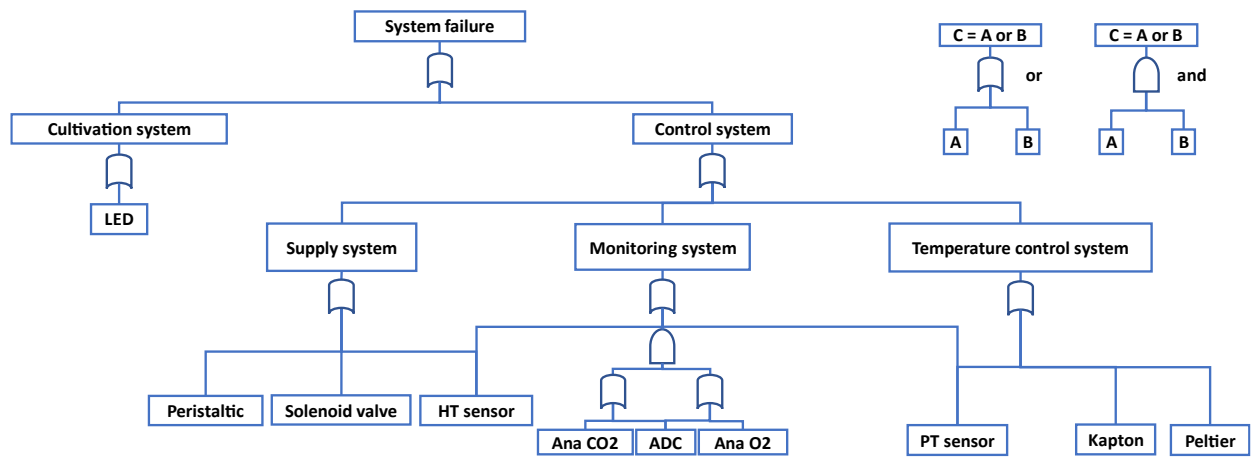

Figure 5 Concept 1 - Fault Tree Analysis 
Yuanchao Ma, Aurelian Vadean, Giovanni Beltrame, Sofiane Achiche

Table 3 Mean time of failure (hours) of each component of Concept 1

\begin{tabular}{|l|l|l|l|}
\hline LED & 50000 & Analog $\mathrm{O}_{2}$ & 43800 \\
\hline Peristaltic & 2300 & ADC & $6.78 \mathrm{E}+07$ \\
\hline Solenoid valve & $2.5 \mathrm{E}+08$ & PT sensor & $3.33 \mathrm{E}+08$ \\
\hline HT sensor & $6.90 \mathrm{E}+08$ & Kapton & 20000 \\
\hline Analog $\mathrm{CO}_{2}$ & 175200 & Peltier & 200000 \\
\hline
\end{tabular}

Given the limited space, the detailed calculation process for each sub-criterion is not presented in this paper but is available in (Ma, 2018). Here we show the calculation process of the criteria RS since it is different from the original MMP. Figure 5 shows the FTA of the entire system of Concept 1 . Table 3 shows the mean time to failure (MTTF) of each component evaluated in Concept 1. The probabilities of failure of each component $\operatorname{Pr}(\mathrm{A})$ and each system $\operatorname{Pr}(\mathrm{C})$ are then calculated by:

$\operatorname{Pr}(A)=1-e^{-\lambda_{A} t}, \lambda_{A}=\frac{1}{M T T F} ; P R(C)=\left\{\begin{array}{l}P R(A)+P R(B), \text { if "or" } \\ P R(A) \times P R(B), \text { if "and" }\end{array}\right.$

with $t=7 \times 24 \times 6 \approx 1000 H$, since the scientific experiment lasts a maximum of 6 weeks. Thus, by considering the equation (4), the $R S_{1}{ }^{*}$ of Concept 1 is calculated by:

$R S_{1}{ }^{*}=\frac{1-\operatorname{Pr}(\text { System Failure })}{\max \left(R S_{i}\right)}=\frac{1-0.09}{0.98}=0.93$

Then, the next step is to obtain the aggregate measurements using the Choquet Integral, which have been applied and proven to be accurate and reliable in multicriteria design problems(Mohebbi et al., 2014, 2018). Thus, the index for evaluating the performance of a system is defined as the global concept score (GCS):

$G C S=C_{\mu}\left(m_{1}^{*}, m_{2}^{*}, m_{3}^{*}, m_{4}^{*}, m_{5}^{*}\right)$

With $C_{\mu}$ representing the Choquet Integral and $m_{i}{ }^{*}$ representing the normalized values of $i^{\text {th }}$ MMP criterion. For the Choquet Integral method, the weighting factor of a subset of criteria is represented by a fuzzy measure on the universe $N$ satisfying the following fuzzy measure equations $\mu()$ :

$\mu(\varnothing)=0, \mu(N)=1, A \subseteq B \subseteq N \rightarrow \mu(A)<\mu(B)$

With $A$ and $B$ representing the fuzzy sets. Thus, GCS can be represented as follows:

$C G S=C_{\mu}\left(m_{1}^{*}, m_{2}^{*}, m_{3}^{*}, m_{4}^{*}, m_{5}^{*}\right)=\sum_{i=1}^{5} \phi(\mu, i) m_{i}^{*}-\frac{1}{2} \sum_{i=1, j=1}^{5} I(\mu, i j)\left|m_{i}^{*}-m_{j}^{*}\right|$

With $\phi(\mu, i)$ representing the importance of criterion $i$ and $I(\mu, i j)$ representing the interaction index between criteria $i$ and $j$. In this study case, these two fuzzy metrics were obtained by questionnaire collection (Ma, 2018) from 10 researchers specializing in nanosatellite system design. They all responded to all 15 fuzzy measures $\phi(\mu, i)=\phi_{i}$ and $I(\mu, i j)=I_{i j}$, and the aggregate results are shown in Table 4 . Table 5 briefly gives the evaluation results for each sub-criterion of the proposed concept. 
Conceptual Design of a Nanosatellite Incubator Using Axiomatic Design and a Mechatronic Multicriteria Profile

Table 4 Fuzzy measurement parameters according to the ten questionnaires

\begin{tabular}{|c|c|c|c|c|}
\hline$\phi_{1}=0.18$ & $\phi_{2}=0.28$ & $\phi_{3}=0.21$ & $\phi_{4}=0.18$ & $\phi_{5}=0.15$ \\
\hline & $I_{12}=0.4$ & $I_{13}=-0.23$ & $I_{14}=0.39$ & $I_{15}=-0.16$ \\
\hline & & $I_{23}=0.57$ & $I_{24}=0.11$ & $I_{25}=-0.20$ \\
\hline & & & $I_{34}=0.22$ & $I_{35}=0.64$ \\
\hline & & & & $I_{45}=-0.30$ \\
\hline
\end{tabular}

Table 5 MMP sub-criteria indices and the GCS of four concepts

\begin{tabular}{|c|c|c|c|c|}
\hline & Concept 1 & Concept 2 & Concept 3 & Concept 4 \\
\hline MIQ & 0.52 & 0.94 & 0.44 & 0.99 \\
\hline RS & 0.93 & 0.96 & 0.91 & 0.97 \\
\hline CX & 0.70 & 0.75 & 0.77 & 0.89 \\
\hline FX & 1.00 & 0.78 & 0.85 & 0.93 \\
\hline CT & 0.59 & 0.68 & 0.94 & 0.63 \\
\hline GCS & 0.58 & 0.80 & 0.61 & $\mathbf{0 . 8 9}$ \\
\hline
\end{tabular}

\section{Results and discussion}

With the GCS in Table 5, we can easily conclude that concept 4 is the "Elite" concept among these four option set choices, with a GCS equal to 0.89 . This system consists of the following parts: two analog gas sensors for $\mathrm{O}_{2}$ and $\mathrm{CO}_{2}$ with two analog-digital converters, a humidity-temperature sensor, a pressure-temperature sensor, thermistors, red and blue LEDs, peristaltic pump with a PWM controller to for flow control.

Furthermore, by looking at Table 4, we can see that reliability and complexity are the two criteria recognized by the ten researchers as relatively important in this design, with $\phi_{2}=$ 0.28 and $\phi_{3}=0.21$. Meanwhile, Concept 4 has the highest RS and CX indices of all the selected concepts. This confirms the results of the questionnaire that a more reliable and less complex design is the ideal design for an incubator that conducts experiments in space. Concept 4 also performs quite well in terms of machine intelligence and flexibility compared to the other concepts. In terms of cost, our design was for a single specific task, so this criterion was considered relatively the least important among the 10 respondents to the questionnaire. Thus, for this type of incubator design, the impact of cost is quite limited within reason.

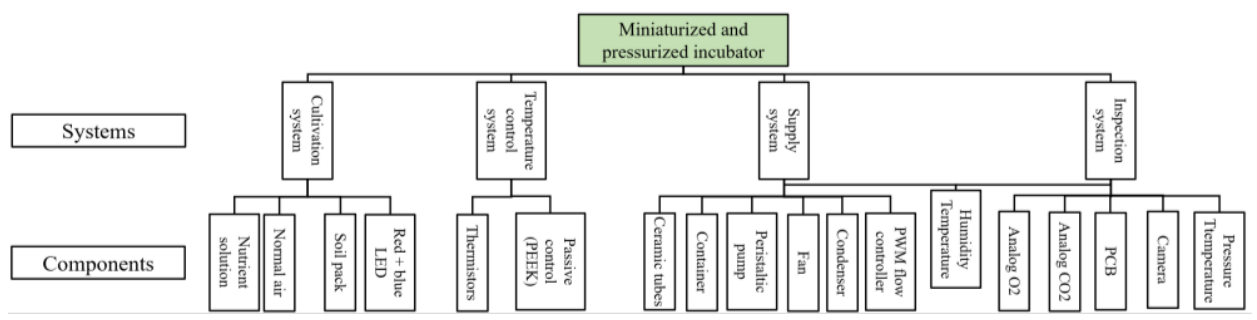

Figure 6 Final system proposed for the conceptual design phase 
Finally, combining the MMP result with the $\mathrm{AD}$ result, the proposed system for the conceptual design phase is shown in Figure 6. Thus, the final concept is a pressurized system consisting of four systems that answer the CAs defined at the beginning:

Cultivation system: this is a pressurized system including normal air (CA2.1). It includes red and blue LEDs as the source of illumination (CA2.3);

Temperature control system: the temperature is actively controlled by the thermistors, and the housing material PEEK is used as passive control to ensure insulation (CA2.4); (3). Supply system: it contains peristaltic pumps and porous tubes for nutrient delivery, as well as fans and mechanical condensers for water vapor recovery. The supply rate is controlled by a PWM controller using data from the humidity sensor. (CA2.2);

Monitoring system: gas concentrations are recorded by analog gas sensors and ADCs. System temperature, humidity, and pressure data are recorded in real time by environmental sensors to control the system and for scientific research. (CA2.5); (5). All components chosen for the design respect the constraints Cs. Based on the results of this conceptual design, the team later performed a detailed layout design (Law-Kam Cio et al., 2021) and built one $1 \mathrm{U}$ proof-of-concept and one $2 \mathrm{U}$ prototype that were successfully tested in plant growth experiments under terrestrial conditions to verify the feasibility of the design (Trouillefou et al., 2021).

\section{Conclusion}

In conclusion, this paper presented the conceptual design of a miniaturized incubator for a biological experiment in a nanosatellite using a synthetic design method using Axiomatic Design and a Mechatronic Multicriteria Profile. Axiomatic Design aids the designer to obtain relevant design attributes through an accurate analysis of project specifications and provides a systematic and hierarchical design approach to derive a portfolio of potential alternatives. Based on this, the Mechatronic Multicriteria Profile index supports the designers to numerically calculate five criteria including machine intelligence, reliability, complexity, flexibility, and production cost. A total of 64 concepts were then evaluated and a Global Concept Score was generated by combining the fuzzy measures and the Choquet Integral. The comparison resulted in an "Elite" solution with a score of 0.89/1. Combined with the results of the Axiomatic Design, a pressurized micro-incubator consisting of four subsystems (cultivation system, temperature control system, supply system and inspection system) was finally obtained.

\section{References}

Bidner, F., 2010. Fault Tree Analysis of the HERMES CubeSat. University of Colorado at Boulder, USA.

Boone, T., Cohen, A., Chin, M., Chinn, T., Friedericks, C., Jackson, E., Keyhan, M., Lera, M., Matin, A., Mayer, D., 2014. E. coli AntiMicrobial Satellite (EcAMSat): Science Payload System Development and Test, Proceedings of the AIAA/USU Conference on Small Satellites,PreConference: CubeSat Developers' Workshop.

ESA, E., 2016. Tailored ecss engineering standards for in-orbit demonstration cubesat projects. ESA, ESTEC: Noordwijk, The Netherlands.

Häuplik-Meusburger, S., Peldszus, R., Holzgethan, V., 2011. Greenhouse design integration benefits for extended spaceflight. Acta Astronautica 68, 85-90.

Hegde, K.M., Abhilash, C., Anirudh, K., Kashyap, P., 2019. Design And Development Of RVSAT1, A Student Nano-satellite With Biological Payload, 2019 IEEE Aerospace Conference. IEEE, pp. 1-14. 
Conceptual Design of a Nanosatellite Incubator Using Axiomatic Design and a Mechatronic Multicriteria Profile

Kramer, H.J., Cracknell, A.P., 2008. An overview of small satellites in remote sensing*. International Journal of Remote Sensing 29, 4285-4337.

Kulak, O., Cebi, S., Kahraman, C., 2010. Applications of axiomatic design principles: A literature review. Expert Systems with Applications 37, 6705-6717.

Law-Kam Cio, Y.-S., Ma, Y., Vadean, A., Beltrame, G., Achiche, S., 2021. Evolutionary layout design synthesis of an autonomous greenhouse using product-related dependencies. Artificial Intelligence for Engineering Design, Analysis and Manufacturing 35, 49-64.

Lee, S., Hutputanasin, A., Toorian, A., Lan, W., Munakata, R., 2009. CubeSat design specification. The CubeSat Program 8651, 22.

Lubián-Arenillas, D., Álvarez, J.M., Bermejo, J., García, S., Cubas, J., Roibás-Millán, E., 2019. Nanosatellite Development Methodology and Preliminary Design Guides for the NANOSTAR Project, 8th European Conference for Aeronautics And Aerospace Sciences (EUCASS), pp. 1-11.

Ma, Y., 2018. Design conceptuel d'un incubateur pour un nanosatellite en combinant la conception axiomatique et un index unifié de performance mécatronique (MMP). École Polytechnique de Montréal.

Mohebbi, A., Achiche, S., Baron, L., 2014. Mechatronic multicriteria profile (MMP) for conceptual design of a robotic visual servoing system, ASME 2014 12th Biennial Conference on Engineering Systems Design and Analysis. American Society of Mechanical Engineers, pp. V003T015A015-V003T015A015.

Mohebbi, A., Achiche, S., Baron, L., 2018. Multi-criteria fuzzy decision support for conceptual evaluation in design of mechatronic systems: a quadrotor design case study. Research in Engineering Design 29, 329-349.

Poghosyan, A., Golkar, A., 2017. CubeSat evolution: Analyzing CubeSat capabilities for conducting science missions. Progress in Aerospace Sciences 88, 59-83.

Rzevski, G., 2014. Mechatronics: Designing intelligent machines Volume 1: Perception, cognition and execution. Newnes.

Stamatelatos, M., Vesely, W., Dugan, J., Fragola, J., Minarick, J., Railsback, J., 2002. Fault tree handbook with aerospace applications. NASA Office of Safety and Mission Assurance, NASA HQ.

Suh, N.P., 1990. The principles of design. Oxford University Press on Demand.

Suh, N.P., Sekimoto, S., 1990. Design of thinking design machine. CIRP Annals-Manufacturing Technology 39, 145-148.

Tang, D.B., Zhang, G.J., Dai, S., 2009. Design as integration of axiomatic design and design structure matrix. Robotics and Computer-Integrated Manufacturing 25, 610-619.

Trouillefou, C.M., Cio, Y.-S.L.-K., Jolicoeur, M., Said, B., Galarneau, A., Achiche, S., Beltrame, G., 2021. An autonomous plant growing miniaturized incubator for a Cubesat. Acta Astronautica $179,439-449$.

Ullman, D.G., 2010. The mechanical design process: Part 1. McGraw-Hill.

Woellert, K., Ehrenfreund, P., Ricco, A.J., Hertzfeld, H., 2011. Cubesats: Cost-effective science and technology platforms for emerging and developing nations. Advances in Space Research 47, 663-684.

Xiang, Z., Wenhe, L., Lianxin, Z., Min, C., Youquan, Y., 2014. Study on on-orbit demonstration technology for space-biology nanosatellites, 2014 International Conference on Manipulation, Manufacturing and Measurement on the Nanoscale (3M-NANO), pp. 271-275.

Zabeau, J.E., N"oel, A., Beltrame, G., 2016. A Miniaturized Incubator Design For Microgravity Botanic Experiments in CubeSats, Proceedings of the 67th International Astronautical Congress.

Contact: Yuanchao Ma, École Polytechnique de Montréal, Mechanical Engineering, C.P. 6079, succ. CV, Montréal, Québec, Canada H3C 3A7, yuanchao.ma@polymtl.ca. 